\title{
Lithium prevents cell apoptosis through autophagy induction
}

\author{
Kazemi $\mathrm{H}^{1}$, Noori-Zadeh $\mathrm{A}^{2}$, Darabi $\mathrm{S}^{3}$, Rajaei $\mathrm{F}^{3}$ \\ Faculty of medicine, Student Research Committee, Qazvin University of Medical Sciences, Qazvin, Iran. \\ shahram2005d@yahoo.com
}

\begin{abstract}
OBJECTIVE: Bone marrow stromal stem cells (BMSCs) are widely used as an available source for cell therapy, tissue engineering, and cellular differentiation-based techniques. Therefore, it is necessary to apply a simple method through which BMSCs can be protected from cell apoptosis under tough conditions of cell differentiation. Lithium treatment is one of the simple methods in this regard.

METHODS: The isolated BMSCs were divided into three groups: (a) control, (b) serum deprivation and (c) LiCl. Cell proliferation and apoptosis and autophagy markers in the presence and absence of $\mathrm{LiCl}$ were evaluated. RESULTS: LiCl has shown to increase survival rate of BMSCs under serum deprivation conditions through autophagy induction (reduced P62 and increased LC3II) and apoptosis inhibition (expression of XIAP), so that the cell survival rate, after 12 hours, was $29 \%, 59 \%, 83 \%, 74 \%, 49 \%$ for the groups, which received 0, 1, $5,10,20$ millimolar of $\mathrm{LiCl}$, respectively, as compared to the control group.

CONCLUSION: LiCl leads to decreased apoptosis and increased survival rate through autophagy induction under serum deprivation conditions (Ref. 5, Ref. 37). Text in PDF www.elis.sk.

KEY WORDS: lithium, bone marrow stromal cells, serum deprivation, autophagy, apoptosis.
\end{abstract}

\section{Introduction}

In recent years, cell therapy $(1,2)$ and gene therapy have gained a considerable attention as a solution for disease treatments $(3,4)$. BMSCs are widely used as an available source for cell therapy and tissue engineering (5). These cells can differentiate into other cell lineages via appropriate induction conditions (5-7). However, these cells quickly disappear after transplantation into the affected area due to serum deprivation, and they usually have low survival rate (8). On one hand, under serum deprivation and starvation conditions, autophagy is activated to protect the cells, but if the serum deprivation is prolonged, apoptosis mechanism will be followed (8). Many studies showed the protective role of lithium against oxidative stress caused by serum deprivation (9-12). Studies also showed that lithium could increase stem cell proliferation $(13,14)$. On the other hand, autophagy is a conserved cellular homeostasis process, which is necessary for eliminating cytoplasmic contents (15). In this study, the role of $\mathrm{LiCl}$ in preventing cell apoptosis through autophagy induction was investigated.

\section{Material and methods}

\section{BMSCs cultivation}

Four adult female Wistar rats (6-8 week old) from Pasteur

${ }^{1}$ Faculty of medicine, Student Research Committee, Qazvin University of Medical Sciences, Qazvin, Iran, ${ }^{2}$ Department of Clinical Biochemistry, Faculty of Paramedicine, Ilam University of Medical Sciences, Ilam, Iran, and ${ }^{3}$ Cellular and Molecular Research Centre, Qazvin University of Medical Science, Qazvin, Iran

Address for correspondence: S. Darabi, Cellular and Molecular Research Centre, Qazvin University of Medical Science, Qazvin, Iran.
Institute were used. Observing ethical rules, the rats were kept in a 12-12 hours light and dark cycle under standard conditions of Qazvin University of Medical Sciences for working with animals. The BMSCs, after being isolated from the long bones of the lower limb and being washed with PBS, were placed in the DMEM/F12 culture media with $10 \% \mathrm{FBS}, 100 \mu \mathrm{g} / \mathrm{mL}$ penicillin, $100 \mathrm{U} / \mathrm{mL}$ streptomycin, $5 \% \mathrm{CO}_{2}$ incubator, $95 \%$ moisture at $37{ }^{\circ} \mathrm{C}$, until the third passage.

\section{Immunocytochemistry}

To investigate the mesenchymal origin of BMSCs at second passage, 5000 cells were cast equally into each 6 -well culture plate. The immunocytochemistry stages were performed as previously mentioned (16). In summary, the cells were placed in paraformaldehyde solution $4 \%$ for 20 minutes and after being washed with phosphate buffered, the cells were placed in $0.3 \%$ Triton X for 15 minutes. After being washed with PBS, the cells were exposed to the primary antibody for 24 hours at $4{ }^{\circ} \mathrm{C}$. The primary antibodies included CD31 (endothelial cells marker), fibronectin (mesenchymal stem cells marker), CD34 (hematopoietic stem cells marker) and P62 (autophagy marker) from ABCAM Company. After the primary antibody incubation, the cells were washed with PBS and then FITC-conjugated secondary antibody (1:100; Chemicon) was added for 2 hours at room temperature. The cells were counted using propidium iodide (PI) through which the nucleus of the cells became red. For assessing autophagy, the BMSCs were immunelabelled with a primary p62 antibody, incubated with the FITCconjugated secondary antibody (shown in green), and a primary LC3II antibody, incubated with Alexa flour secondary antibody (shown in red). The number of autophagosome immune-reactive cells was determined to estimate the autophagy activity. 


\section{Cell proliferation}

Cell Counting Kit-8 (CCK8) was used to examine the effect of $\mathrm{LiCl}$ on cellular proliferation of BMSCs. The cells were divided into the two groups: (a) BMSCs and (b) BMSCs $+\mathrm{LiCl}$. The test was performed on the cells at days $0,1,3$ and 5 , and then, cell proliferation was compared. In summary, the cells were first seeded on a 96-well culture plate, and after performing the tests on the cell groups, the CCK 8 solution was added to the cells, which were incubated for one hour, and finally, the optical density of the cells was measured at $450 \mathrm{~nm}$ by an ELISA reader.

\section{Viability test}

In order to examine the effects of serum deprivation and the protective role of $\mathrm{LiCl}$, viability test was performed by trypan blue staining. 5000 cells were seeded into a 96-well plate, into three groups: (a) control; (b) serum deprivation; (c) serum deprivation (SD) with $\mathrm{LiCl}$. The control group consisted of BMSCs with no treatments. The second group was serum-deprived, in which the BMSCs placed in the serum-free medium without $\mathrm{LiCl}$ (BMSCs+ $\mathrm{SD})$. The third group belonged to serum deprivation and $\mathrm{LiCl}$ (BMSCs $+\mathrm{SD}+\mathrm{LiCl}$ ), in which the cells were exposed to $\mathrm{LiCl}$ at different doses $(1,5,10$ and $20 \mathrm{MM})$ for 12 hours. The mortality rates in the cell groups were evaluated using trypan blue staining viability test using a Neobar lam under the microscope. The percentage of living cells can be obtained by counting the whole cells and stained cells. Each cell group was counted three times under the microscope.

\section{ELISA test}

The cells of different groups were investigated in terms of the expression of XIAP using ELISA. The proteins were extracted by lysing the cells in RIPA buffer (Sigma) and kept at $-80{ }^{\circ} \mathrm{C}$. The XIAP expressions evaluated according to the company's protocols (i.e., R\&D Systems, Minneapolis, MN).

\section{Statistical analysis}

The results were analysed using SPSS software version 21 (SPSS Inc., Chicago, IL, USA) and analysis of variance (ANOVA) with Tukey's multiple tests for comparing among groups.

\section{Results}

\section{BMSCs cultivation}

The cells adhered to the bottom of the flask after 48 hours and the spindle-shaped cells filled about $80 \%$ of the flask bottom after 12 hours. At this stage, the cells maintained their spindleshaped fibroblastic appearance (Fig. 1A). HSCs (hematopoietic stem cells) and other existing cells in bone marrow were cleared off after being washed.

\section{Cluster of differentiation cell markers immunocytochemistry}

The cells attached to the bottom of the flask were evaluated by immunocytochemistry in terms of CD cell markers. They were positive in terms of fibronectin (bone marrow stem cell marker) (Fig. 1B) and negative in terms of CD31 (endothelial cell marker)
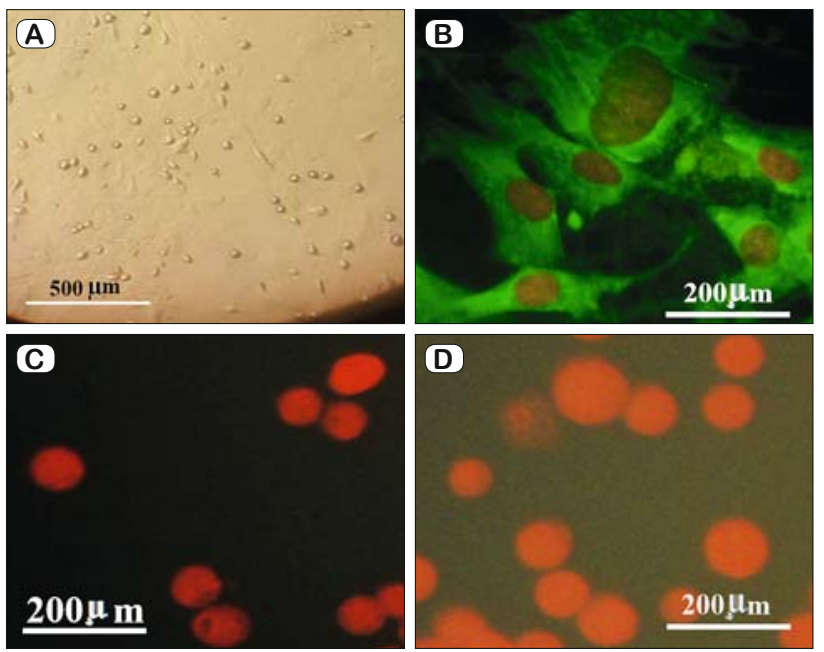

Fig. 1. Immunostaining of BMSCs for different markers of cell differentiation. A) BMSCs at third passage. B) Fibronectin. C) CD31, D) CD34. BMSCs were immunolabeled with primary antibody, incubated with the FITC-conjugated secondary antibody, and counter-stained using propidium iodide (PI).

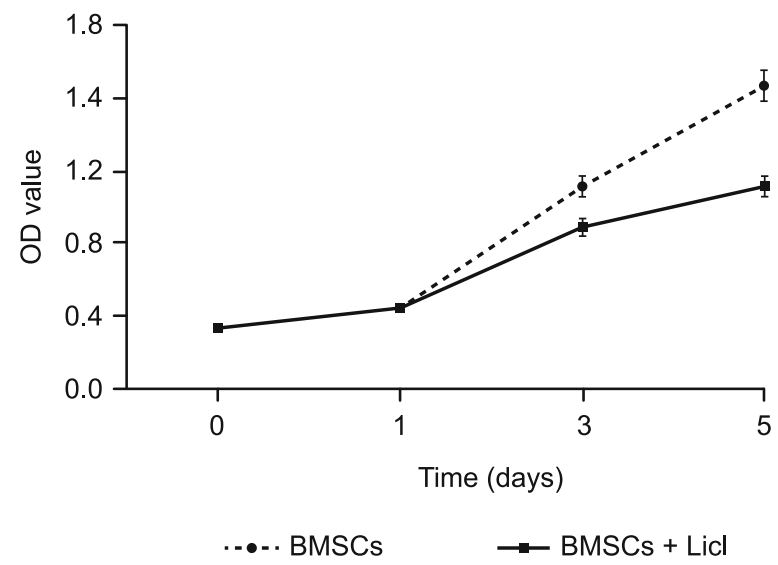

Fig. 2. Proliferation was analysed by the CCK-8 assay. The proliferation of BMSCs was significantly increased by $\mathrm{LiCl}(* \mathrm{p}<0.05)$.

(Fig. 1C) and CD34 (HSCs marker) (Fig. 1D). The green colour of the fluorescence light is related to the conjugated secondary antibody to the FITC. The cells were counterstained using propidium iodide (PI), while the cell nucleus emitted red fluorescent light.

\section{Cell proliferation by $\mathrm{LiCl}$}

Cell proliferation test was performed by CCK- 8 on two group of BMSCs (control) and BMSCs+ LiCl. It was observed that the number of the cells increased after being placed in $5 \mathrm{MM}$ of $\mathrm{LiCl}$ for 48 hours. On the fifth day, the difference in cell proliferation for the $\mathrm{BMSCs}+\mathrm{LiCl}$ group was significantly higher than that of the BMSCs group ( $\mathrm{p}<0.05)$, and 1.5 times increase was observed for the BMSCs+ $\mathrm{LiCl}$ group compared to the BMSCs group. In this study, the number of the BMSCs was significantly increased after exposing to $\mathrm{LiCl}$ at day 5 , compared to the control group (Fig. 2). 


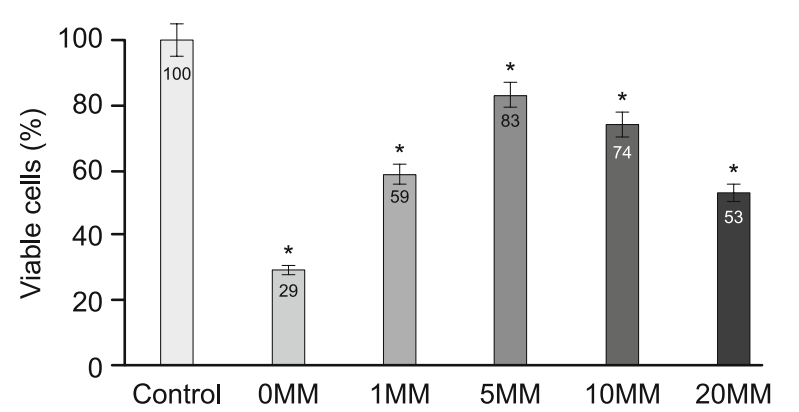

Fig. 3. Cell viability by trypan blue in BMSCs treated without (control) or with $\mathrm{LiCl}(0,1,5,10,20 \mathrm{mM}) .(* \mathrm{p}<0.05)$ compared to control groups.

\section{BMSCs viability}

Viability test was performed on the cells in order to examine the protective effect of the $\mathrm{LiCl}$ under serum deprivation conditions to prevent the BMSCs death. In the viability test, the untreated BMSCs were considered as the control group in third passage. In order to evaluate the protective effects of the $\mathrm{LiCl}$, the cells were treated without serum at different concentrations of the $\mathrm{LiCl}$ for 12 hours. After 12 hours, the cell viability was $29 \%, 59 \%, 83$ $\%, 74 \%, 49 \%$, respectively, for the group, which received 0,1 , 5,10 and $20 \mathrm{MM}$ of $\mathrm{LiCl}$, compared to the control group. The mortality rate was higher for the group, which did not receive $\mathrm{LiCl}$ and serum (survival rate: $29 \%$, viability: below $50 \%$ ) and significant differences $(\mathrm{p}<0.05)$ were observed compared to the control group (Fig. 3).

\section{LiCl in autophagy induction}

In order to examine whether oxidative stress caused by serum deprivation can affect autophagosome formation, the BM$\mathrm{SCs}$ with/without serum were exposed to the $\mathrm{LiCl}$ (5 MM) for 12 hours (Fig. 4). The level of autophagy was evaluated by determining LC3II/LC3I and P62. The P62 is an autophagy marker. It can be bound to the LC3 and then can be degraded through autophagy-lysosomal pathway. Therefore, high level of the P62 indicates a problem in autophagy. In BMSCs (control) in normal autophagy, P62 is at a low level and LC3II was not expressed (Fig. 4A). In this study, it was observed that P62 was increased by reducing serum level (serum deprivation or starvation state) and increasing time, in a sense that the highest level of P62 observed in the cells without treatment with $\mathrm{LiCl}$ after 12 hours indicating autophagy inhibition (Fig. 4C). The level of LC3II after 12 hours of serum deprivation was reduced (Fig. 4C). However, this ratio increased in an environment containing $\mathrm{LiCl}$ (5 MM) (Fig. 4B). Quantification of the number of autophagosomes per cell in BM$\mathrm{SCs}$, BMSCs $+\mathrm{SD}$, and BMSCs $+\mathrm{SD}+\mathrm{LiCl}$ groups have been shown in Figure 4D. The autophagosome number of BMSCs+ SD group increased twofold in comparison with the BMSCs $+\mathrm{SD}+$ $\mathrm{LiCl}$ group (Fig. 4D).

\section{LiCl effects on XIAP protein levels}

In the BMSCs (control) group, the expression of XIAP was $4 \mathrm{ng} / \mu \mathrm{l}$, which was reached to $1 \mathrm{ng} / \mu \mathrm{l}$ in BMSCs + SD group. However, XIAP was higher for the group BMSCs $+\mathrm{SD}+\mathrm{LiCl}$ with $12 \mathrm{ng} / \mu \mathrm{l}$ (Fig. 5).

\section{Discussion}

The BMSCs are widely used as an invaluable source of cell therapy. Therefore, it is necessary to apply a simple method through which BMSCs number can be increased and cell apoptosis can be prevented in serum deprivation conditions without using growth factors and genetic engineering methods. Lithium treatment is one of the simple methods in this regard. Interestingly, after removing $\mathrm{LiCl}$ from the BMSCs culture medium, it was observed that cell proliferation returned to its original state. These reversible effects were already observed in the nervous system. For example, in a study, it was found that lithium neurotoxicity disappeared after its discontinuation since lithium could easily cross the plasma membrane and did not bind to the membrane proteins, thus, it was easily expelled by the kidneys $(17,18)$. So, using Li as a
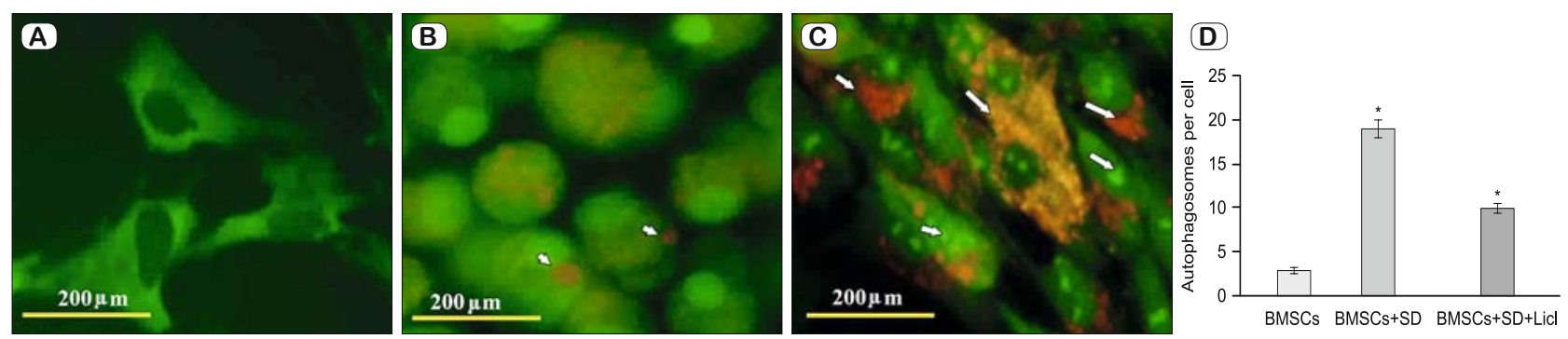

Fig. 4. Immunohistochemical localization of $\mathrm{p} 62$ and LC3II in BMSCs. LC3II and P62 accumulate in BMSCs. (A) P62 and LC3 immunostaining of BMSCs (control). Note the accumulations of P62 (green) in the BMSCs. (B) LiCl stimulation of autophagy in BMSCs. In bone marrow stromal Starved cells (serum deprived) treated with the $\mathrm{LiCl}(5 \mathrm{mM})$ for $12 \mathrm{~h}$, Autophagosomes appear as LC3 and/or P62 puncta. Arrows denote vacuoles or accumulations of P62 (green) or LC3II (red). P62 and LC3II-immunoreactive inclusions seemed to be near each other, but they did not absolutely colocalize; (C) Distribution of autophagosome types according to the expression of LC3II and P62. Autophagosomes expressing P62 are shown in green and LC3II alone are shown in red, respectively; autophagosomes co-expressing LC3II and P62 are shown in yellow. BMSCs were immunolabeled with the primary P62 antibody, incubated with FITC- conjugated secondary antibody (green), and primary LC3 antibody, incubated with the Alexa flour secondary antibody (red). (D) Quantification of the number of autophagosomes per cell is shown in $\mathrm{D} .{ }^{*} \mathbf{p}<\mathbf{0 . 0 5}$, for the differences between the indicated groups with BMSCs (control) groups. 


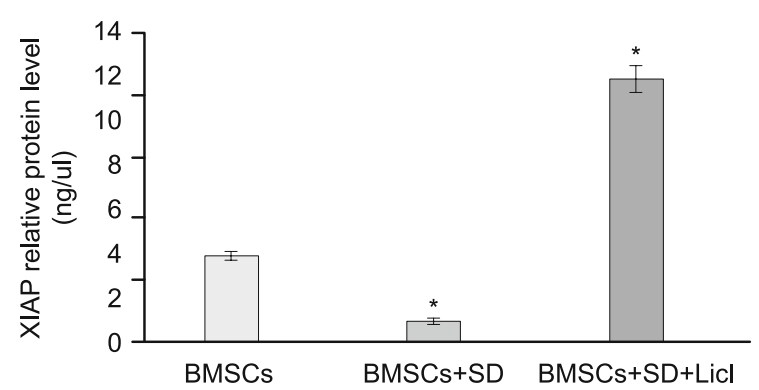

Fig. 5. XIAP relative protein expression in BMSCs, BMSCs + SD, and BMSCs + SD+ LiCl groups. XIAP protein level regulated by serum deprivation (SD) and $\mathrm{LiCl}$ in BMSCs. * $\mathrm{p}<0.05$, for the differences among the indicated groups.

therapeutic drug is advantageous, in this regard. In this study, (a) $\mathrm{LiCl}$ increased BMSCs proliferation without affecting the phenotype and differentiation of them, (b) $\mathrm{LiCl}$ prevented BMSCs from apoptosis through autophagy induction under serum deprivation conditions. For assessing anti-apoptotic effects of $\mathrm{Li}$ on the cells, we used X chromosome-linked inhibitor of apoptosis (XIAP) as a marker, as it is one of the anti-apoptotic proteins, which inhibit the activity of the cell death proteases, caspase-3, -7, and -9 (19, $20)$. The BIR2 domain of the XIAP can inhibit caspase- $3,-7$ while BIR3 is needed for potent caspase-9 inhibition (20). Therefore, XIAP has a great potential to inhibit apoptosis in BMSCs under serum deprivation. In this study, we indicated that in BMSCs, the level of XIAP was reduced under serum deprivation conditions and increased in the environment containing $\mathrm{LiCl}$. Lithium can reduce apoptosis by increasing the expression of XIAP. Studies showed that lithium could increase the expression of Bcl-2 and heat shock protein while reducing the expression of proapoptotic p53 and $\operatorname{Bax}(21)$. Lithium can reduce the activity of caspase 3 and calpain (22). Lithium inhibits the activity of glycogen synthase kinase-3b (GSK-3b). GSK-3b has a protective role; therefore, the cell can be protected when GSK-3b is inhibited. Under normal nutritional conditions, TRIP-Br3 can increase the survival rate of the cells through fixing XIAP. However, under starvation and serum deprivation conditions, it is degraded, which ultimately results in XIAP protein being unstable and the cell dies. XIAP can inhibit caspases in the cell by activating the mitochondria antioxidant enzymes. It has been previously reported that the increased expression of XIAP had the ability to prevent the transplanted cells from apoptosis under hypoxia and inflammatory conditions. Previous studies showed that XIAP could prevent apoptosis caused by caspases and viral infections. The XIAP can inhibit and destroy caspases through activating proteasome (23). The XIAP anti-apoptotic properties were reported to have decreased effect in HL60 cells under serum deprivation conditions (24). Thus, XIAP alone cannot prevent apoptosis per se and other factors must be taken into consideration.

The results indicated that lithium could lead to autophagy induction by converting LC3I to LC3II and reducing P62. Although the appropriate functions of lithium are known, its mechanism in cell survival through activating and augmenting autophagy was not well-known. In BMSCs, the mortality rate was increased as the serum level of culture medium was reduced in the presence of lithium. In this study, we indicated that lithium could increase the cell survival rate through autophagy induction under serum deprivation conditions. The conversion of LC3-I to LC3-II represents autophagy in mammals. P62 can be broken down by autophagy and acts as a carrier receptors (cargo) in order to destroy ubiquinone substrates. P62 can be bound and aggregated with LC3/Atg8 through LIR, LC3-Interacting Region. Recent studies indicated that LC3-I is converted to LC3-II due to a food deprivation, reduced oxygen and chemotherapy and is placed into an autophagosome membrane, and therefore, LC3-II in cell would lead to autophagy induction. In hypoxia or serum deprivation conditions, as well as stressful conditions for the cell, the proteins and damaged organelles can be degraded and recycled through the process of autophagy. LC3-II along with P62 participates in the formation of autophagosomes membranes. LC3 can be found in Soluble LC3I and LC3-II is its lipidated and autophagosome form. In other words, LC3-II is a conjugated form of LC3. During autophagy, autophagosome takes cytoplasmic components containing proteins and cytosolic organelles. Subsequently, cytosolic form of LC3 (LC3-I) binds to phosphatidylethanolamine (PE) in order to create conjugated LC3-PE (LC3-II), which is used in autophagosome membrane (25). We analysed the sequestosome-1 (SQSTM1/ P62), here referred to as P62, to investigate the Li effects on the autophagy process as it is an important intracellular protein, which is induced by cellular stress and plays a significant role in regulating the signalling pathways of the cell death. It is a scaffold protein containing many parts functioning in signal transmission, proliferation, cell survival, death, swelling, tumour formation, and the response to oxidative stress. P62 can clear misfolded/aggregated proteins as well as damaged organelles in cells by regulating autophagy (26). P62 can promote mammalian target of rapamycin complex 1 (mTORC1) activity and inhibit autophagy, by binding to mTORC1 and phosphorylating Unc-51 like Autophagy Activating Kinase (ULK) 1/2 and, therefore, reduced P62 would deactivate $\mathrm{mTORC} 1$ and activate autophagy (27). On the other hand, microtubule-associated proteins $1 \mathrm{~A} / 1 \mathrm{~B}$ light chain $(\mathrm{LC} 3) 3 \mathrm{~B}$ is a protein that in humans is encoded by the MAP1LC3B gene and is used to assess autophagy activity levels as we used it in the current study. The LC3 is one of the important proteins in the autophagy pathway, where it functions in substrate selection and autophagosome biogenesis. The LC3 can be found in the cytosol as LC3I form, while LC3II is concentrated in autophagosome membrane (28). In many studies, the expression of LC3 was investigated as an indicator of autophagy induction as the previous study reported (15). Moreover, the conversion of LC3I to LC3II represents autophagy initiation, and greater amounts of LC3II indicate an increase in the formation of autophagic vacuoles in the cell. Autophagosomes bind to lysosomes and create an autolysosome and its contents are degraded by lysosomal hydrolysis. In accordance with our study, also previous studies showed the protective effects of lithium in autophagy induction (29) in oligodendrocytes (30), microglia (31), endothelial cells (32), dopaminergic neurons (33), cerebel- 
lar cells (34), and PC12 cells (9). In addition, lithium also plays a protective role in autophagy induction in many diseases such as: spinal cord injury (35), amyotrophic lateral sclerosis, prion, Parkinson's disease and colorectal cancer (36). The previous study showed that lithium protected PC12 cells under serum deprivation conditions by activating the PI3K/Akt pathway and inhibiting FoxO1 (9). Many studies showed the protective effects of lithium and these effects attributed to the activating phosphatidylinositol 3-kinase/Akt4 and the extracellular signal-regulated kinase (ERK) pathway (37). However, in this regard, we focused on the autophagy-mediated actions of lithium.

\section{Conclusion}

$\mathrm{LiCl}$ leads to reducing apoptosis and augmenting autophagy, proliferation and survival rate of the cells under serum deprivation conditions.

\section{References}

1. Haratizadeh S, Nazm Bojnordi M, Darabi S, Karimi N, Naghikhani M, Ghasemi Hamidabadi $\mathbf{H}$ et al. Condition medium of cerebrospinal fluid and retinoic acid induces the transdifferentiation of human dental pulp stem cells into neuroglia and neural like cells. Anat Cell Biol 2017; 50 (2): $107-114$.

2. Noori-Zadeh A, Mesbah-Namin S A, Bistoon-Beigloo S, Bakhtiyari S, Abbaszadeh H A, Darabi S et al. Regulatory T cell number in multiple sclerosis patients: A meta-analysis. Mult Scler Relat Disord 2016; 5: 73-76.

3. Darabi S, Tiraihi T, Delshad A, Sadeghizadeh M, Taheri T, Hassoun H K. Creatine Enhances Transdifferentiation of Bone Marrow Stromal Cell-Derived Neural Stem Cell Into GABAergic Neuron-Like Cells Characterized With Differential Gene Expression. Mol Neurobiol 2017; 54 (3): 1978-1991.

4. Noori-Zadeh A, Mesbah-Namin S A, Tiraihi T, Rajabibazl M, Taheri T. Non-viral human proGDNF gene delivery to rat bone marrow stromal cells under ex vivo conditions. Journal of the neurological sciences 2014; 339 (1-2): 81-86.

5. Naghdi P, Tiraihi T, Ganji F, Darabi S, Taheri T, Kazemi H. Survival, proliferation and differentiation enhancement of neural stem cells cultured in three-dimensional polyethylene glycol-RGD hydrogel with tenascin. J Tissue Eng Regen Med 2016; 10 (3): 199-208.

6. Darabi S, Tiraihi T, Ruintan A, Abbaszadeh H A, Delshad A, Taheri T. Polarized neural stem cells derived from adult bone marrow stromal cells develop a rosette-like structure. In Vitro Cell Dev Biol Anim 2013; 49 (8): 638-652.

7. Darabi S, Tiraihi T, Delshad A, Sadeghizadeh M. A new multistep induction protocol for the transdifferentiation of bone marrow stromal stem cells into GABAergic neuron-like cells. Iran Biomed J 2013; 17 (1): 8-14.

8. Zhang Q, Yang Y J, Wang H, Dong Q T, Wang T J, Qian H Y et al. Autophagy activation: a novel mechanism of atorvastatin to protect mesenchymal stem cells from hypoxia and serum deprivation via AMPactivated protein kinase/mammalian target of rapamycin pathway. Stem Cells Dev 2012; 21 (8): 1321-1332.

9. Zeng Z, Wang H, Shang F, Zhou L, Little P J, Quirion R et al. Lithium ions attenuate serum-deprivation-induced apoptosis in PC12 cells through regulation of the $\mathrm{Akt} / \mathrm{FoxO} 1$ signaling pathways. Psychopharmacology (Berl) 2016; 233 (5): 785-794.

10. Schmidt M M, Guan K, Wobus A M. Lithium influences differentiation and tissue-specific gene expression of mouse embryonic stem (ES) cells in vitro. Int J Dev Biol 2001; 45 (2): 421-429.

11. Wieck A. Prevention of bipolar episodes with lithium in the perinatal period. Br J Psychiatry 2017; 211 (1): 3-4.

12. Young W. Review of lithium effects on brain and blood. Cell Transplant 2009; 18 (9): 951-975.

13. Zhu Z, Yin J, Guan J, Hu B, Niu X, Jin D et al. Lithium stimulates human bone marrow derived mesenchymal stem cell proliferation through GSK-3beta-dependent beta-catenin/Wnt pathway activation. FEBS J 2014; 281 (23): 5371-5389.

14. Dong B T, Tu G J, Han Y X, Chen Y. Lithium enhanced cell proliferation and differentiation of mesenchymal stem cells to neural cells in rat spinal cord. Int J Clin Exp Pathol 2015; 8 (3): 2473-2483.

15. Shams Nooraei M, Noori-Zadeh A, Darabi S, Rajaei F, Golmohammadi Z, Abbaszadeh H A. Low Level of Autophagy-Related Gene 10 (ATG10) Expression in the 6-Hydroxydopamine Rat Model of Parkinson's Disease. Iran Biomed J 2018; 22 (1): 15-21.

16. Darabi S T T, Noori-Zadeh A, Rajaei F, Darabi L, Abbaszadeh HA. Creatine and retinoic acid effects on the induction of autophagy and differentiation of adipose tissue-derived stem cells into GABAergic-like neurons J Babol Univ Med Sci 2017; 19 (8): 41-49.

17. Netto I, Phutane V H. Reversible lithium neurotoxicity: review of the literatur. Prim Care Companion CNS Disord 2012; 14 (1):

18. de Cates A N, Morlet J, Antoun Reyad A, Tadros G. Lithium overdose and delayed severe neurotoxicity: timing for renal replacement therapy and restarting of lithium. BMJ Case Rep 2017; 2017

19. Deveraux Q L, Takahashi R, Salvesen G S, Reed J C. X-linked IAP is a direct inhibitor of cell-death proteases. Nature 1997; 388 (6639): 300-304.

20. Unsain N, Higgins J M, Parker K N, Johnstone A D, Barker P A. XIAP regulates caspase activity in degenerating axons. Cell Rep 2013; 4 (4): 751-763.

21. Chen R W, Chuang D M. Long term lithium treatment suppresses p53 and Bax expression but increases Bcl-2 expression. A prominent role in neuroprotection against excitotoxicity. J Biol Chem 1999; 274 (10): 6039-6042.

22. Crespo-Biel N, Camins A, Pallas M, Canudas A M. Evidence of calpain/cdk5 pathway inhibition by lithium in 3-nitropropionic acid toxicity in vivo and in vitro. Neuropharmacology 2009; 56 (2): 422-428.

23. Li C, Jung S, Lee S, Jeong D, Yang Y, Kim K I et al. Nutrient/serum starvation derived TRIP-Br3 down-regulation accelerates apoptosis by destabilizing XIAP. Oncotarget 2015; 6 (10): 7522-7535.

24. Notarbartolo M, Cervello M, Dusonchet L, Cusimano A, D'Alessandro N. Resistance to diverse apoptotic triggers in multidrug resistant HL60 cells and its possible relationship to the expression of Pglycoprotein, Fas and of the novel anti-apoptosis factors IAP (inhibitory of apoptosis proteins). Cancer Lett 2002; 180 (1): 91-101.

25. Schaaf M B, Keulers T G, Vooijs M A, Rouschop K M. LC3/GABARAP family proteins: autophagy-(un)related functions. FASEB J 2016; 30 (12): 3961-3978.

26. Feng Y, Klionsky D J. Autophagy regulates DNA repair through SQSTM1/p62. Autophagy 2017; 13 (6): 995-996. 
27. Duran A, Amanchy R, Linares J F, Joshi J, Abu-Baker S, Porollo A et al. p62 is a key regulator of nutrient sensing in the mTORC1 pathway. Mol Cell 2011; 44 (1): 134-146.

28. Yang A, Hacheney I, Wu Y W. Semisynthesis of autophagy protein LC3 conjugates. Bioorg Med Chem 2017; 25 (18): 4971-4976.

29. Motoi Y, Shimada K, Ishiguro K, Hattori N. Lithium and autophagy. ACS Chem Neurosci 2014; 5 (6): 434-442.

30. Del Grosso A, Antonini S, Angella L, Tonazzini I, Signore G, Cecchini M. Lithium improves cell viability in psychosine-treated MO3.13 human oligodendrocyte cell line via autophagy activation. J Neurosci Res 2016;c94 (11): 1246-1260.

31. Fabrizi C, Pompili E, Somma F, De Vito S, Ciraci V, Artico M et al. Lithium limits trimethyltin-induced cytotoxicity and proinflammatory response in microglia without affecting the concurrent autophagy impairment. J Appl Toxicol 2017; 37 (2): 207-213.

32. Kim E C, Meng H, Jun A S. Lithium treatment increases endothelial cell survival and autophagy in a mouse model of Fuchs endothelial corneal dystrophy. Br J Ophthalmol 2013; 97 (8): 1068-1073.
33. Hou L, Xiong N, Liu L, Huang J, Han C, Zhang G et al. Lithium protects dopaminergic cells from rotenone toxicity via autophagy enhancement. BMC Neurosci 2015; 16: 82.

34. Chang J W, Choi H, Cotman S L, Jung Y K. Lithium rescues the impaired autophagy process in $\mathrm{CbCln} 3$ (Deltaex 7/8/Deltaex 7/8) cerebellar cells and reduces neuronal vulnerability to cell death via IMPase inhibition. J Neurochem 2011; 116 (4): 659-668.

35. Liu P, Zhang Z, Wang Q, Guo R, Mei W. Lithium Chloride Facilitates Autophagy Following Spinal Cord Injury via ERK-dependent Pathway. Neurotox Res 2017.

36. Wood H. Neurodegenerative disease: Lithium promotes accumulation of brain iron via tau suppression. Nat Rev Neurol 2016; 12 (9): 492-493.

37. Xia Y, Wang C Z, Liu J, Anastasio N C, Johnson K M. Lithium protection of phencyclidine-induced neurotoxicity in developing brain: the role of phosphatidylinositol-3 kinase/Akt and mitogen-activated protein kinase kinase/extracellular signal-regulated kinase signaling pathways. J Pharmacol Exp Ther 2008; 326 (3): 838-848.

Received December 23, 2017. Accepted January 19, 2018. 